\title{
A systematic review of psychosocial suicide prevention interventions for youth
}

\author{
Alison L. Calear ${ }^{1} \cdot$ Helen Christensen ${ }^{2} \cdot$ Alexander Freeman $^{3} \cdot$ Katherine Fenton $^{3}$. \\ Janie Busby Grant ${ }^{4} \cdot$ Bregje van Spijker $^{1}$ Tara Donker ${ }^{5,6}$
}

Received: 16 July 2015 / Accepted: 5 October 2015

(C) Springer-Verlag Berlin Heidelberg 2015

\begin{abstract}
Youth suicide is a significant public health problem. A systematic review was conducted to examine the effectiveness of school, community and healthcarebased interventions in reducing and preventing suicidal ideation, suicide attempts and deliberate self-harm in young people aged 12-25 years. PsycInfo, PubMed and Cochrane databases were searched to the end of December 2014 to identify randomised controlled trials evaluating the effectiveness of psychosocial interventions for youth suicide. In total, 13,747 abstracts were identified and screened for inclusion in a larger database. Of these, 29 papers describing 28 trials fulfilled the inclusion criteria for the current review. The results of the review indicated that just over half of the programs identified had a significant effect on suicidal ideation (Cohen's $d=0.16-3.01$ ), suicide attempts (phi $=0.04-0.38)$ or deliberate self-harm (phi $=0.29$ $0.33 ; d=0.42$ ). The current review provides preliminary
\end{abstract}

Alison L. Calear

alison.calear@anu.edu.au

1 National Institute for Mental Health Research, The Australian National University, 63 Eggleston Road, Acton, ACT 2601, Australia

2 Black Dog Institute, University of New South Wales, Hospital Road, Randwick, NSW 2031, Australia

3 Australian National University Medical School, 54 Mills Road, Acton, ACT 2601, Australia

4 University of Canberra, University Drive, Bruce, ACT 2617, Australia

5 Department of Clinical Psychology, VU University, van der Boechorststraat 1, 1081 BT Amsterdam, The Netherlands

6 EMGO Institute for Health and Care Research, VU University and VU University Medical Center, van der Boechorststraat 7, 1081 BT Amsterdam, The Netherlands support for the implementation of universal and targeted interventions in all settings, using a diverse range of psychosocial approaches. Further quality research is needed to strengthen the evidence-base for suicide prevention programs in this population. In particular, the development of universal school-based interventions is promising given the potential reach of such an approach.

Keywords Suicide $\cdot$ Prevention $\cdot$ Early intervention · School $\cdot$ Community $\cdot$ Healthcare

\section{Introduction}

Suicide is a significant public health problem and one of the leading causes of death in young people [1]. In 2010 and 2012, the suicide rate for people aged 15 to 24 years was 10.5 per 100,000 people in the United States and Australia, respectively [2, 3]. Suicidal ideation and suicide attempts are also a major concern. In a systematic review of suicide phenomena in young people worldwide, the mean proportion of adolescents reporting a lifetime suicide attempt was $9.7 \%$, while $29.9 \%$ reported suicidal thoughts [4]. The societal and fiscal burden associated with suicidal behaviour (ideation, attempts and completion) is also high, and includes emotional and psychosocial morbidity, medical care, lost productivity and the secondary distress caused to family members and friends [5].

The prevalence of youth suicide, and the significant burden accompanying it, has given rise to the development of a range of psychosocial interventions aimed at preventing and reducing suicidal behaviour and promoting help-seeking and early identification of suicide in young people [6, 7]. The need to promote and assist help-seeking behaviour among youth is critical, as young people often do not seek 
or receive help for suicidal thoughts and behaviour $[6,8-$ 10]. Interventions for youth suicide prevention have been implemented in schools, communities and healthcare systems, and are designed to reduce risk factors for suicidal behaviour, or to identify individuals at risk and provide pathways to treatment or support $[6,7]$. Psychosocial suicide prevention programs have been delivered individually, or in groups, and have tended to be based on common therapeutic approaches, such as cognitive behaviour therapy (CBT), dialectical behaviour therapy (DBT) and problem solving therapy [6].

Depending on the approach employed, these programs can be delivered universally or to a selective or indicated population. Universal programs are offered to all young people in a particular setting (e.g., classroom program) and are designed to enhance protective factors or reduce risk factors across a whole population [11, 12]. Selective interventions are targeted to young people identified as being "at risk" of suicide, while indicated programs are designed for young people already exhibiting suicidal behaviour, such as suicidal ideation or attempt [7,11-13].

Unlike road safety, which has a clear framework to reduce mortality and morbidity, suicide prevention to a large extent lacks a preventative, strategic framework. To some degree this is due to a lack of a strong evidence-base around potentially effective strategies in a range of settings. Previous reviews $[6,7,13-16]$ have looked at youth suicide and self-harm prevention programs in isolation according to a specific setting (e.g., schools, clinical settings), but to our knowledge no recent evaluation has considered the overall evidence-base. The objective of the present review therefore is to identify randomised controlled trials (RCTs) of psychosocial interventions for youth suicide in school, community and healthcare settings, with the aim of identifying what types of interventions can be effective in these settings and where future research efforts should be directed.

\section{Methods}

\section{Identification and inclusion of studies}

The trials identified in the current review are drawn from a large database of psychosocial interventions for suicidal ideation, plans and attempts maintained by the Australian National Health and Medical Research Council (NHMRC) Centre of Research Excellence in Suicide Prevention (CRESP [17]). This database is based on searches conducted in PsycInfo, PubMed, and Cochrane up to December 31st 2014, with the key search terms "Suicid* OR self-harm OR self-poisoning AND trial OR intervention OR prevention”. Figure 1 presents a flowchart detailing the review process. The titles and abstracts of the 13,747 articles identified in the searches were screened by two reviewers to determine their inclusion in the database. Completely irrelevant articles that were unrelated to the topic of the database (i.e., did not discuss suicide or prevention) were immediately excluded, while potentially relevant studies and reviews were retained and the full-text article examined for inclusion. Additional articles were also obtained from reference list searches of previous systematic reviews and meta-analyses.

The inclusion criteria for the database were that: (a) the program trialed was a psychosocial intervention for the treatment or prevention of suicidal behaviour, (b) suicidal behaviour (self-harm, ideation, attempt or completion) was a primary or a secondary outcome measure for the trial, (c) the study was a randomised controlled trial with a no intervention, wait-list, attention or treatment as usual (TAU) control condition, and (d) the trial was published in a peerreviewed, English language journal [17]. Psychosocial interventions were defined as programs providing psychotherapy (e.g., CBT, DBT, problem solving therapy), psychoeducation or community treatment or support. Trials of pharmacological interventions were only included if they contained a psychosocial comparison. For this database, all suicide and related constructs were included as outcome measures. The terms used to describe these outcomes were drawn from the descriptions provided by the paper authors. These terms were "deliberate self-harm", "suicide ideation", "suicide attempts", and "suicidality" (ideation, plans, attempts, deliberate self-harm). Although self-harm may not involve suicide intent, there is evidence that it may lead to suicidal behaviour $[18,19]$.

Studies were excluded if the intervention examined was not designed to specifically address suicide or self-harm. It was outside of the scope of this review to include interventions for other disorders that might include a suicide outcome measure. Studies were also excluded if the intervention did not directly target or intervene with the population of interest. This included screening studies, in which participants were only referred to outside services if they screened positive, and gate-keeper interventions that did not include outcome data on the at-risk population (e.g., only reported improvements in the trained workforce). Suicide interventions that were not evaluated within a RCT framework, or did not include suicide-related outcome measures, were also excluded to ensure that the highest level of research evidence could be captured in the review [17]. No restrictions were placed on intervention setting or method of delivery (e.g., individual vs. group, face-to-face vs. distal).

For the current review, trials from the database were included if study participants were adolescents or young adults aged between 12 and 25 years. Studies that 
Fig. 1 Study identification flow diagram

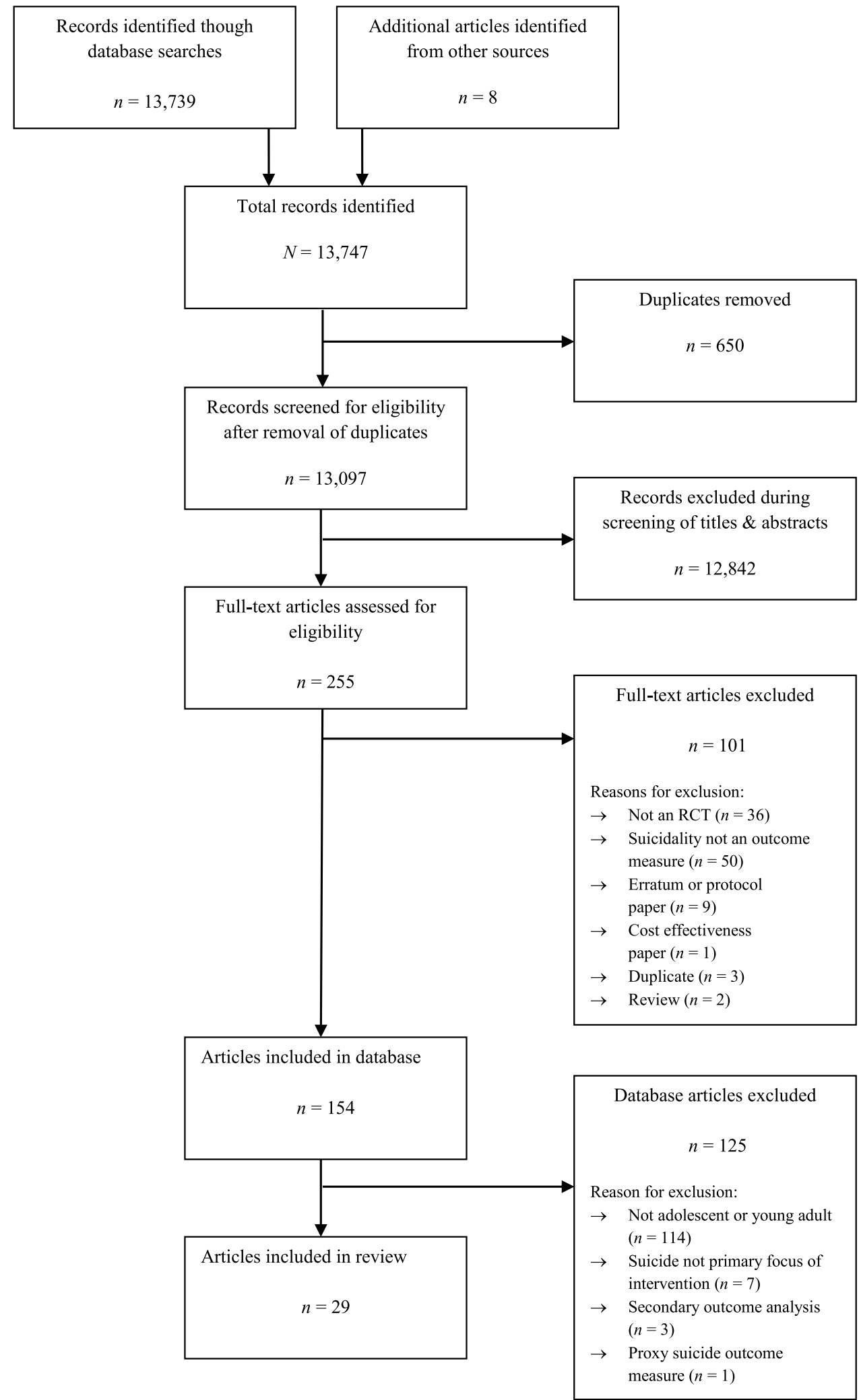


employed a proxy measure of suicide risk (e.g., elevated levels of anxiety and depression) were also excluded from the current review.

\section{Data extraction}

Papers that fulfilled the inclusion criteria were coded by two independent researchers, with all relevant data extracted (see [17]). Disagreements between reviewers were resolved through discussion with a third researcher. Where data were available and extractable, standardised between groups effect size (ES) estimates were calculated using Cohen's $d$ [20] or phi. Cohen's $d$ is calculated by subtracting the mean intervention $(\mathrm{Mi})$ score from the mean control score $(\mathrm{Mc})$ and dividing by the pooled standard deviation $[(\mathrm{Mc}-\mathrm{Mi}) / \mathrm{SD}]$. Positive standardised effect size estimates indicate that the intervention group improved more than the control group. According to Cohen [20], an effect size of 0.20 is considered small, while 0.50 is considered moderate and 0.80 is considered large. Phi was used to calculate the effect size of studies with a dichotomous outcome variable and was obtained by dividing the Chi square statistic by the sample size, and then taking the square root of the result [21]. An effect size of 0.10 is considered small, 0.30 moderate and 0.50 large [21]. A formal meta-analysis was not conducted as the pooling of studies was not appropriate given the vast differences in participant characteristics, interventions and measurement of outcomes. For ease of reporting summary statistics, trials reporting multiple measurement occasions will be deemed "effective" if a significant difference between the intervention and control condition is reported on at least one measurement occasion. Effect sizes will be reported for all measurement occasions.

The quality of each study was also assessed using four criteria from the "risk of bias" assessment tool [22], which was developed by the Cochrane collaboration. This quality rating tool assesses possible sources of bias in randomized controlled trials. The four criteria assessed in the current review included the adequate generation of allocation sequence; the concealment of allocation to conditions; the prevention of knowledge of condition allocation (masking of assessors); and dealing with incomplete outcome data (this was assessed as positive when intention-to-treat analyses were conducted). Quality ratings were only based on the information reported in the included trial paper. Additional publications or study authors were not consulted.

\section{Results}

Overall, 29 relevant papers were identified in the review, describing 28 individual trials of a suicide intervention program for young people. Three of the trials [all evaluating the school-based Counselors Care, Assess, Respond, Empower (C-CARE), Coping and Support Training (CAST) and/or Parents Care, Assess, Respond, Empower (P-CARE) programs] involved multiple intervention arms with a control condition. As such, there are 32 possible outcome comparisons.

\section{Trial characteristics}

Table 1 presents the characteristics of each trial, as well as their outcomes for suicidal ideation, suicide attempts and/ or deliberate self-harm. Of the 32 comparisons identified in the current review, $10(31 \%)$ reported on a program delivered in a school-based setting, seven $(22 \%)$ on a program in a community (non-clinical) setting (e.g., homebased, distal) and $15(47 \%)$ on an intervention delivered within a healthcare (clinical) setting (e.g., in-patient hospital, health centre). A total of 10,654 participants were recruited across all studies. Study sample sizes ranged from 30 to 4133 participants (median $=108$ participants). The reported mean age of participants ranged from 12.9 to 22 years (median $=15.6$ years, $n=25$ ), while the percentage of male participants in each trial ranged from 10 to $82 \%$ (median $=31.9 \%, n=30$ ). Of the 32 comparisons, $22(69 \%)$ evaluated an indicated intervention in which participants who had a history of suicidal ideation or attempt were recruited to the trial, while eight $(25 \%)$ were selective (elevated risk due to history of depression or deliberate self-harm) and two (6\%) were universal. Participants were recruited from a variety of settings, including schools (34 \%), in-patient facilities (28\%), outpatient clinics (28\%), emergency departments (25\%), universities $(6 \%)$ and primary care $(6 \%)$. A range of psychosocial interventions were implemented in the trials identified. These included social support (37\%), CBT (25\%), problemsolving therapy (22\%), motivational interviewing (22\%), psychoeducation (13\%) and DBT (9\%).

Twenty-seven ( $84 \%$ ) of the 32 comparisons evaluated a face-to-face intervention only, while three (9\%) studies tested a distal intervention using postcards, tokens or a video, and two (6\%) studies reported a combined face-toface and telephone-based intervention. Program delivery was diverse, with 12 (37\%) of the 32 comparisons evaluating an individual program alone, seven $(22 \%)$ reporting on a combined individual and family/parent program, six $(19 \%)$ on a group program, four $(13 \%)$ on a family program, two $(6 \%)$ on an individual and group program and one $(3 \%)$ on a parent-only program. Twenty-four $(75 \%)$ of the 32 comparison programs were structured and had a set number of sessions, while the remaining eight $(25 \%)$ programs had varied program lengths that differed between participants. Among the structured programs, the number of sessions ranged from 1 to 64 (median $=5, n=24$ ). 


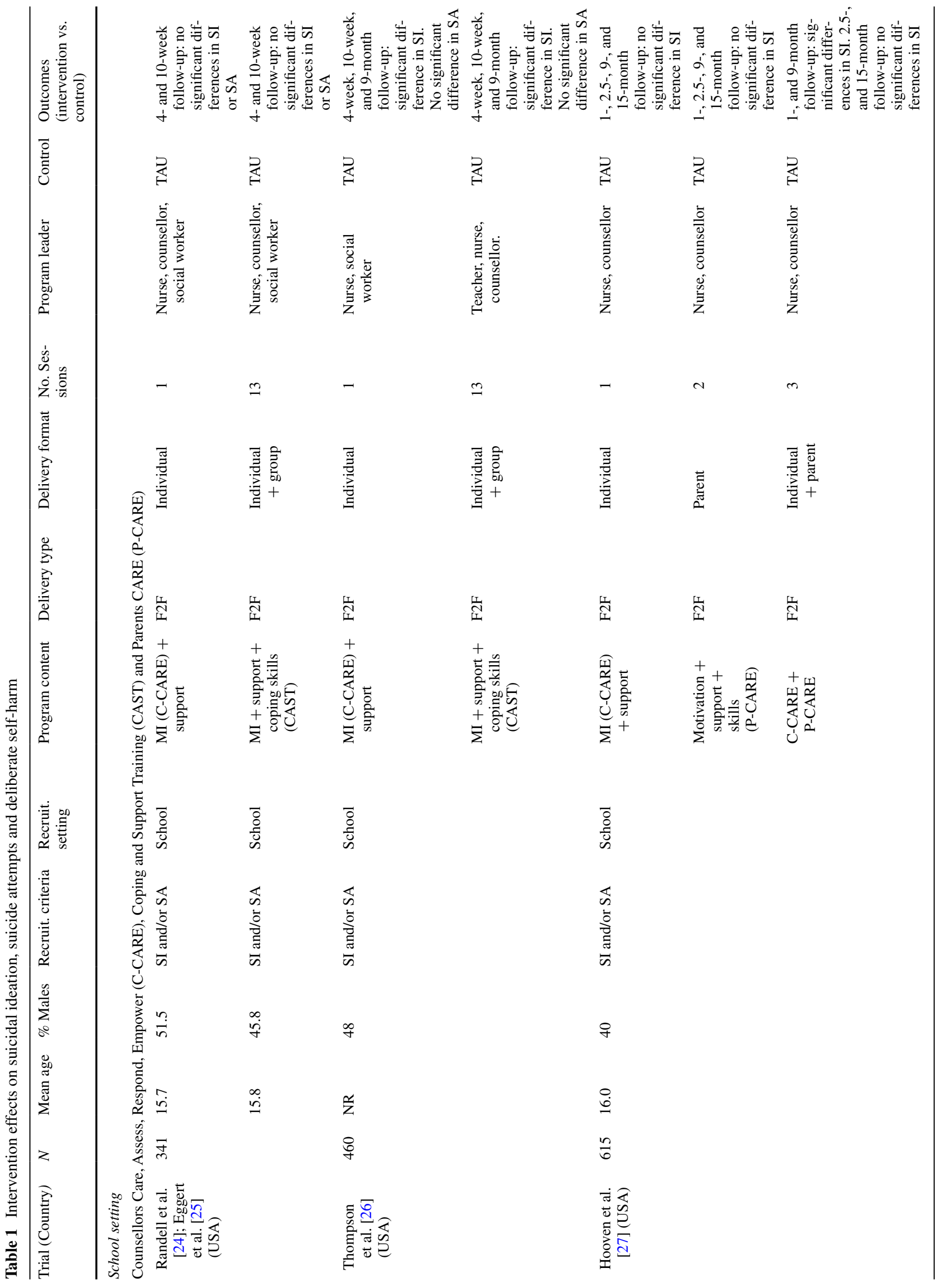




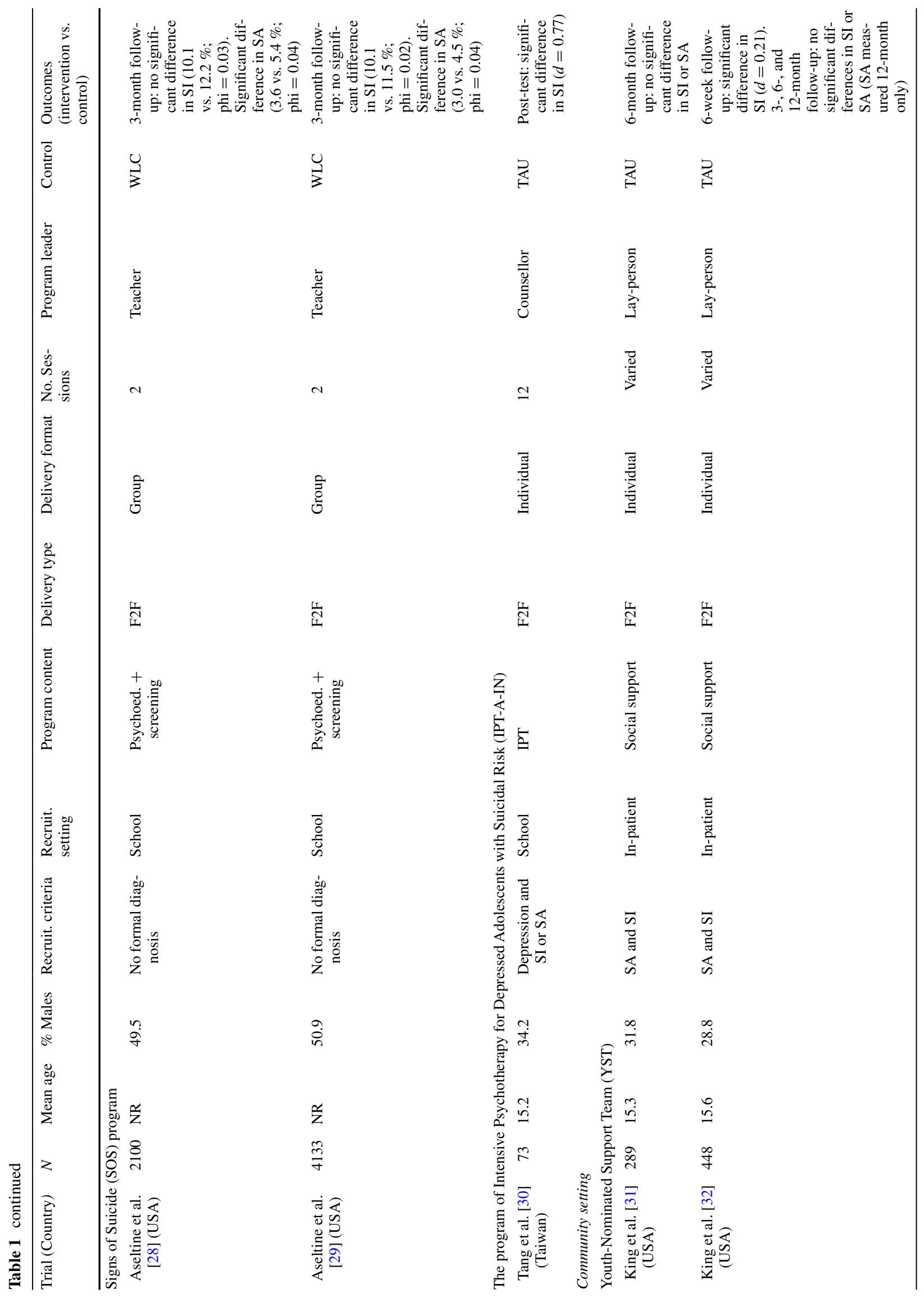




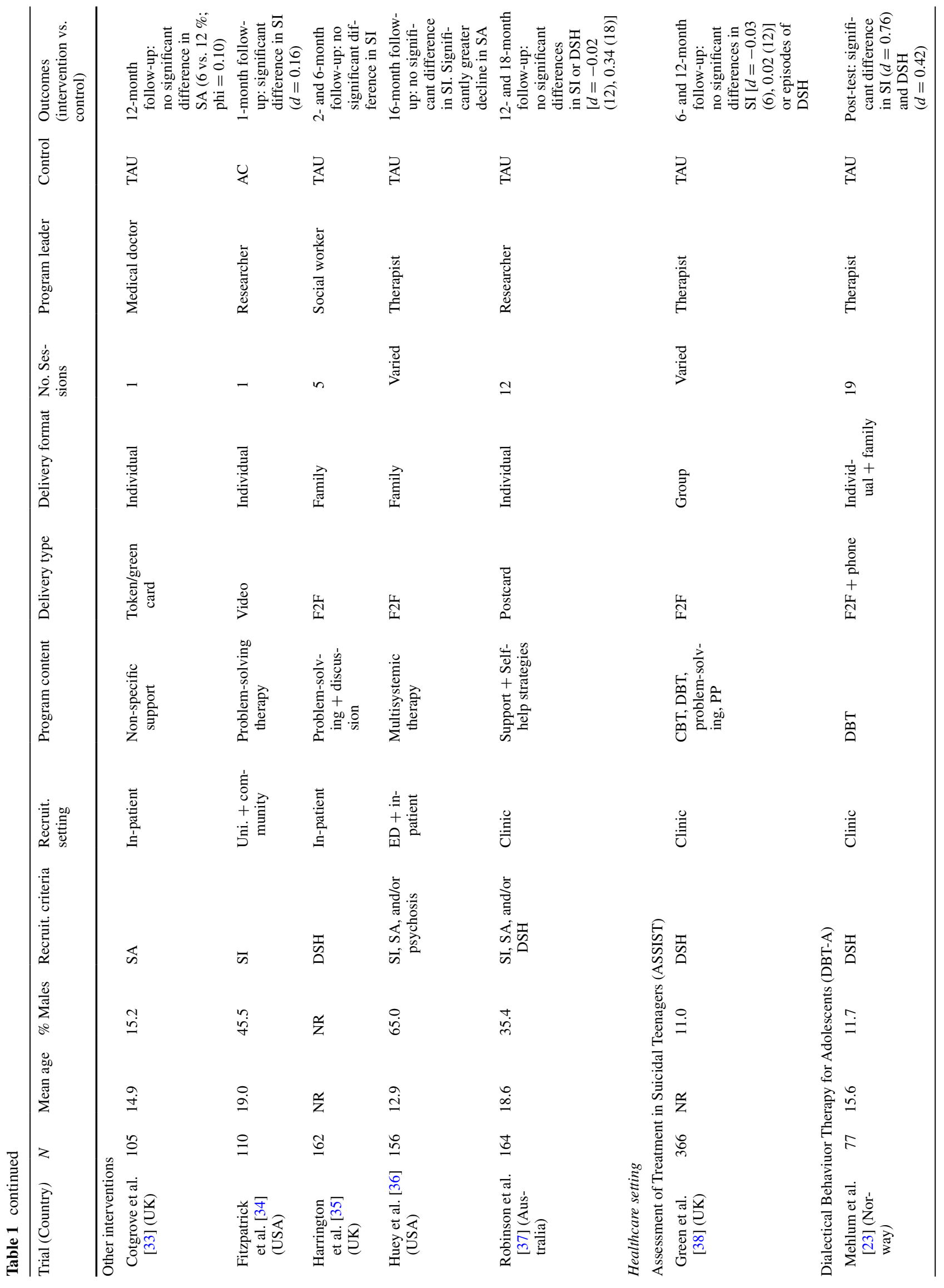




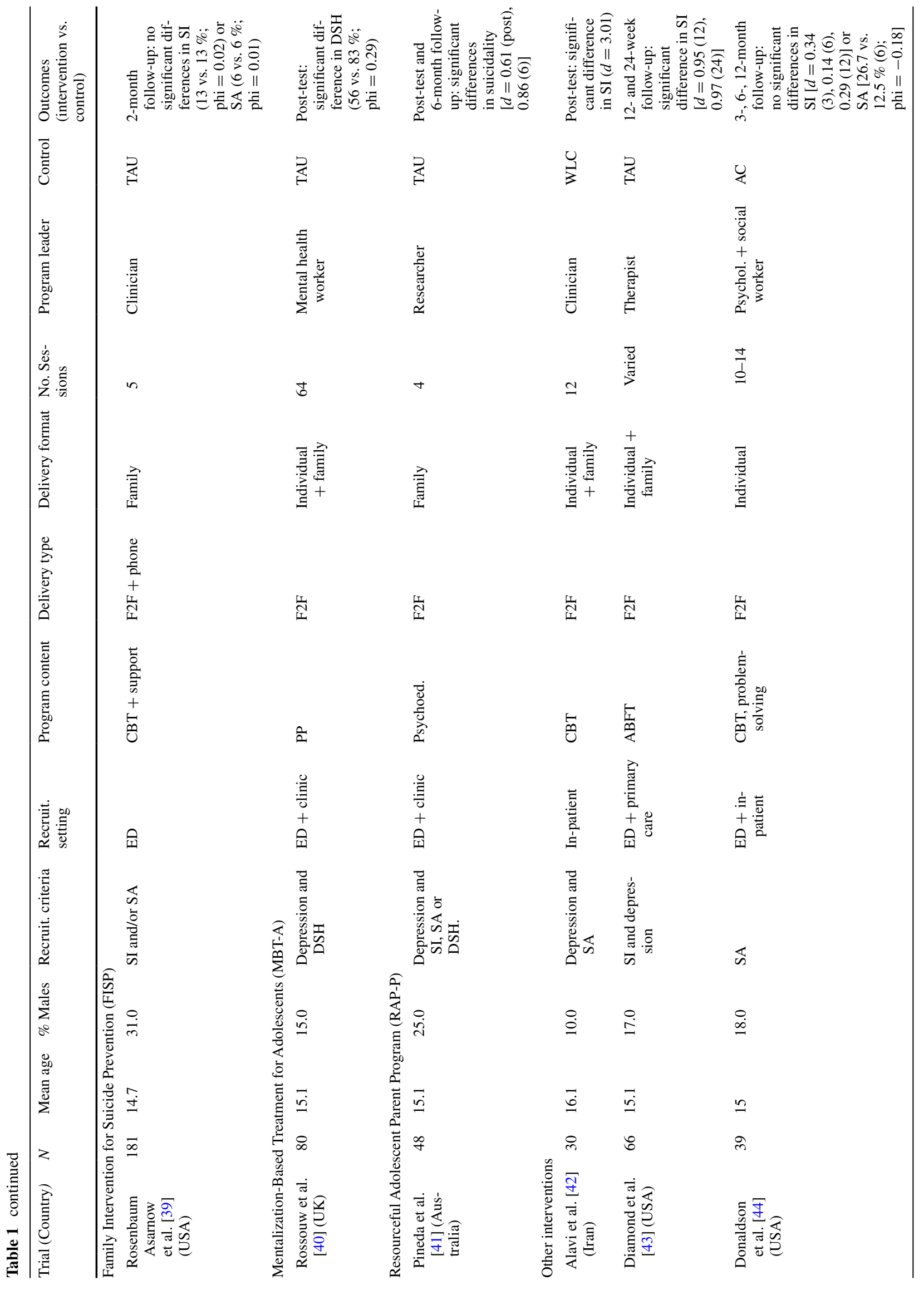




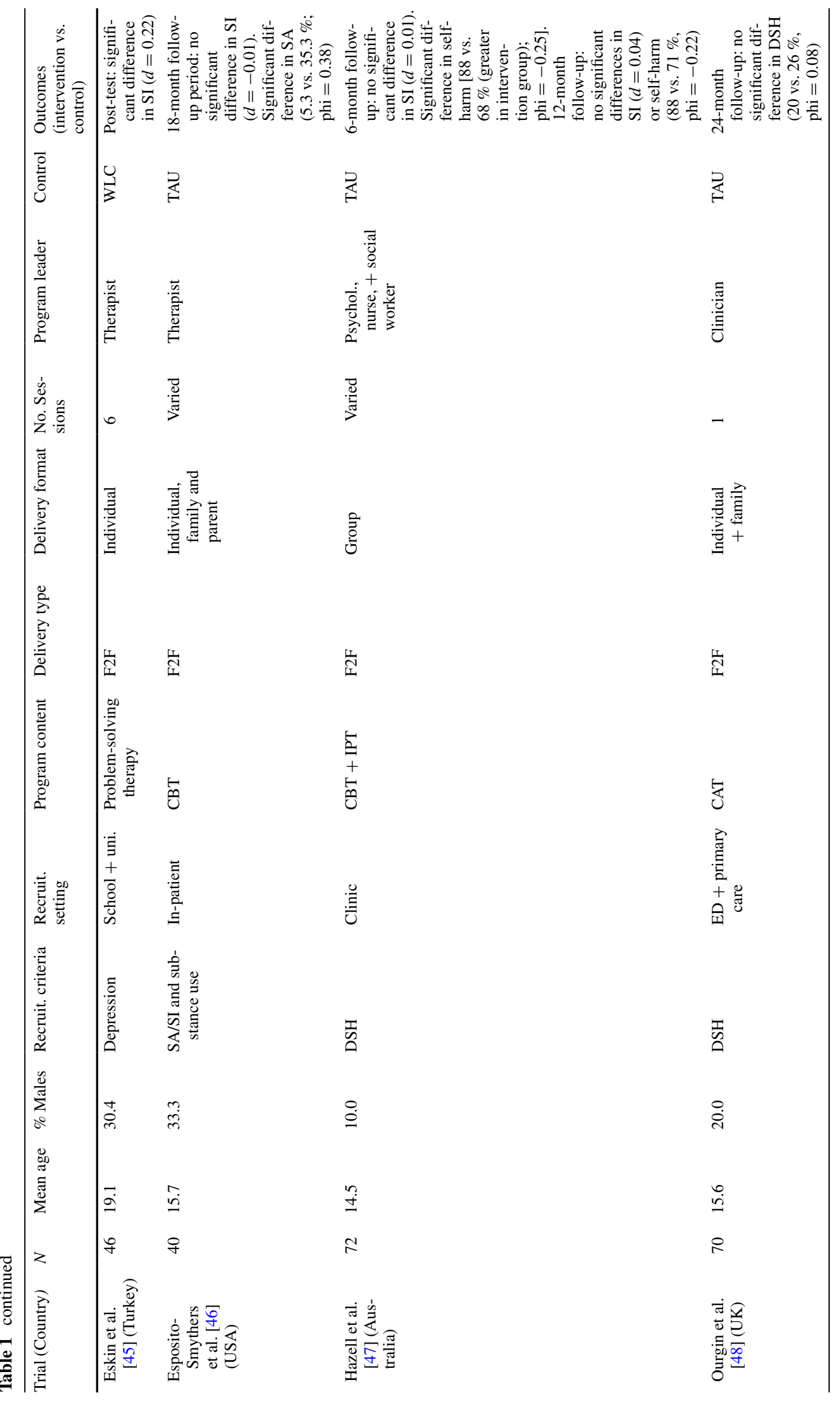




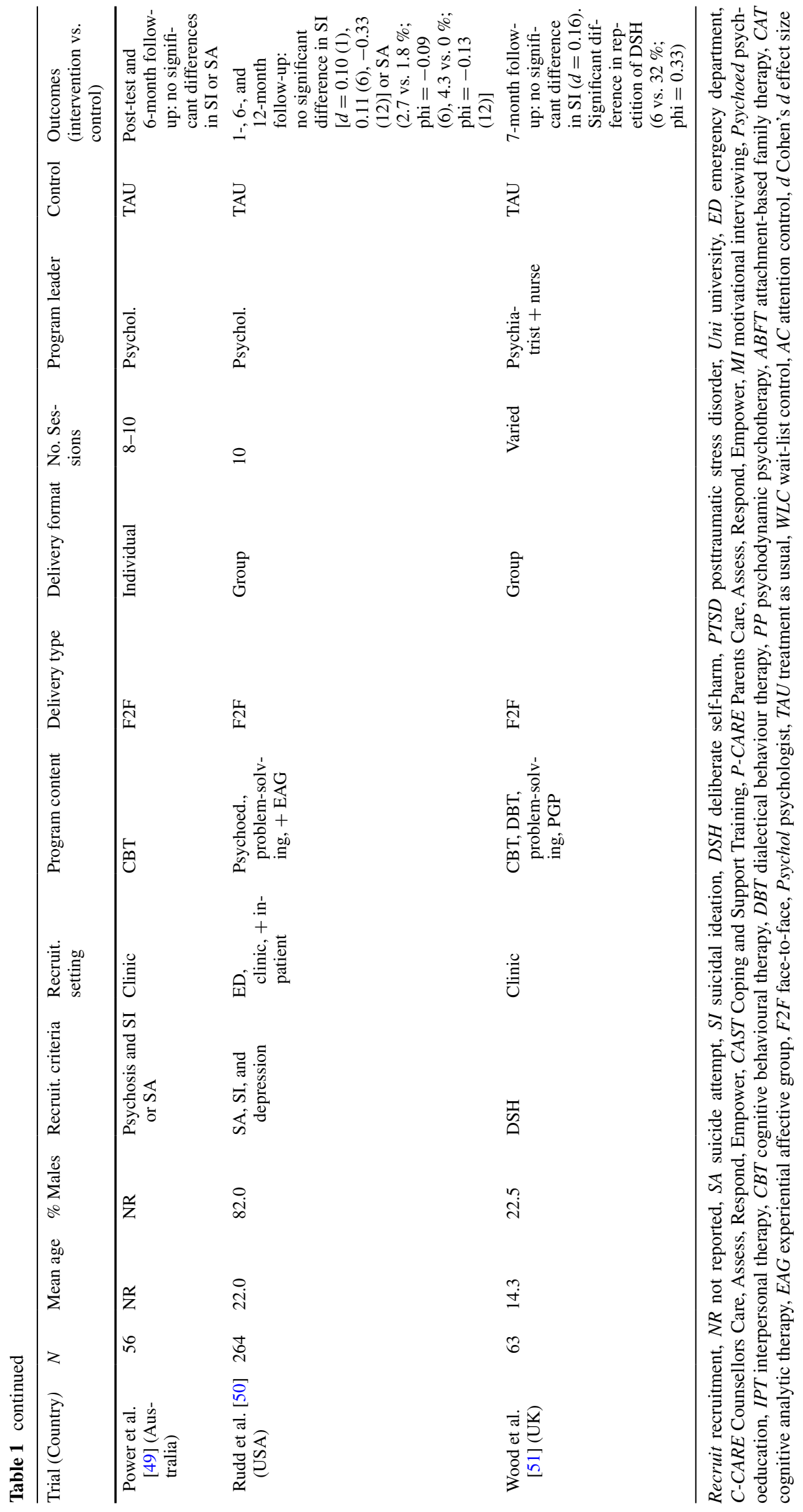


Interventions often included more than one program leader, which included nurses $(28 \%)$, social workers $(19 \%)$, therapists $(19 \%)$, psychologists $(13 \%)$ and teachers $(9 \%)$. Twenty-six $(81 \%)$ of the 32 comparisons included a treatment as usual control condition, four $(12 \%)$ included a wait-list control condition and two $(6 \%)$ utilised an attention control condition.

\section{General findings}

Overall, 17 of the $32(53 \%)$ comparisons reported a significant effect in favour of the intervention condition on suicidal ideation, suicide attempts, deliberate self-harm (DSH), and/or suicidality at immediate post-intervention or follow-up. More specifically, 28 of the 32 ( $88 \%$ ) outcome comparisons reported on intervention effects for suicidal ideation. Of these 28 comparisons, 10 (36\%) found significant differences between the intervention and control conditions $(d=0.16-3.01$, median $=0.76, n=7)$, and $18 \mathrm{did}$ not $(d=-0.33$ to 0.34 , median $=0.07, n=6$; phi $=0.02-$ 0.03 , median $=0.02, n=3$ ). In terms of intervention effects on suicide attempts, 15 of the possible 32 (47\%) outcome comparisons measured suicide attempts following the intervention. Four of the $15(27 \%)$ comparisons reported a significant effect on suicide attempts $(\mathrm{phi}=0.04-0.38$, median $=0.04, n=3$ ), while 11 comparisons reported no effect (phi $=-0.18$ to 0.10 , median $=-0.09, n=4$ ). Seven of the 32 (22\%) comparisons reported on intervention effects for deliberate self-harm (DSH). Of these seven comparisons, three $(43 \%)$ found significantly lower levels of DSH following the intervention ( $\mathrm{phi}=0.29-0.33$, $n=2 ; d=0.42, n=1)$, three (43\%) reported no significant effects ( $d=-0.02$ to $0.34, n=1$; phi $=0.08, n=1)$, and one study reported a significant effect in favour of the control condition (phi $=-0.25$ ). Finally, one of the 32 (3\%) comparisons included suicidality as an outcome measure and found significant differences $(d=0.61-0.86$, $n=1$ ). Only one study [23] reported positive effects on multiple outcomes measures (suicidal ideation and deliberate self-harm).

\section{Effect of intervention setting}

Six of the 17 (35\%) effective programs were delivered in the school environment, with four of the programs reporting a significant difference in suicidal ideation $(d=0.77$, $n=1)$ and two finding differences in suicide attempts (phi $=0.04, n=2$ ). Three of the $17(18 \%)$ effective programs were presented in a community setting, of which two had an effect on suicidal ideation $(d=0.16-0.21$, median $=0.19, n=2$ ) and one reported a significant difference in suicide attempts. The remaining eight $(47 \%)$ effective interventions were delivered in a healthcare setting, with four programs resulting in differences in suicidal ideation $(d=0.22-3.01$, median $=0.87, n=4)$, one reporting significant effects on suicide attempts $(\mathrm{phi}=0.38$ ), three having an effect on DSH ( $\mathrm{phi}=0.29-0.33, n=2$; $d=0.42, n=1)$, and one on suicidality $(d=0.61-0.86)$. Overall, $60 \%$ of the school-based programs identified in the review were effective, $43 \%$ of the community-based interventions and $53 \%$ of the programs delivered in a healthcare setting.

\section{Effect of intervention content}

Ten of the 17 (59\%) effective programs delivered a psychotherapeutic intervention (e.g., CBT, problem solving therapy), with six of the programs reporting a significant difference in suicidal ideation $(d=0.16-3.01$, median $=0.77, n=6$ ), two programs finding differences in suicide attempts (phi $=0.38, n=1)$ and three reporting a positive intervention effect on deliberate self-harm (phi $=0.29-0.33, n=2 ; d=0.42, n=1$ ). The remaining seven effective programs contained less formal psychosocial interventions, such as social support, psychoeducation and motivational interviewing. Of these effective programs, four had an effect on suicidal ideation $(d=0.21, n=1)$, two reported differences in suicide attempts ( $\mathrm{phi}=0.04$, $n=2$ ) and one reported significant changes in suicidality $(d=0.61-0.86)$. Overall, $55 \%$ of the programs that included a traditional psychotherapeutic approach were effective and $50 \%$ of programs containing another type of psychosocial intervention reported a positive effect.

\section{Effect of intervention approach}

Two of the 17 (12\%) effective programs were universally delivered, with both of these programs reporting significant effects on suicide attempts (phi $=0.04, n=2$ ). A further four $(23 \%)$ of the effective programs were delivered selectively to participants with a history of depression or deliberate self-harm, of which two found a significant effect on suicidal ideation $(d=0.22-0.76, n=2)$ and three an effect on deliberate self-harm ( $\mathrm{phi}=0.29-0.33, n=2 ; d=0.42$, $n=1)$. The remaining 11 (65\%) effective programs were delivered to an indicated population with a history of suicidal ideation or attempts, of which eight reported significant differences in suicidal ideation $(d=0.16-3.01$, median $=0.77, n=5$ ), two had an effect on suicide attempts (phi $=0.38, n=1$ ), and one reported changes in suicidality $(d=0.61-0.86, n=1)$. Overall, all of the universal programs identified in the current review were found to be effective, as were $50 \%$ of the selective programs and $50 \%$ of the indicated programs identified. 


\section{Effect of delivery format}

Five of the 17 (29\%) effective programs were delivered to the individual alone, with all of these programs reporting positive effects on suicidal ideation $(d=0.16-0.77$, $n=4)$. Three of the $17(18 \%)$ effective programs were group-based, and had significant effects on suicide attempts (phi $=0.04-0.33, n=3$ ). A further six (35\%) of the programs were a combined individual and parent/family intervention, of which four reported a significant difference in suicidal ideation $(d=0.76-3.01$, median $=0.97, n=3)$, one reported effects on suicide attempts (phi $=0.38$ ), and two reported changes in DSH (phi $=0.29, n=1$; $d=0.42, n=1$ ). The remaining three effective programs were two family based programs and a combined individual and group-based intervention. The family-based programs reported effects on suicide attempts or suicidality ( $d=0.61-0.086, n=1)$, while the individual and groupbased intervention had an effect on suicidal ideation. Overall, $86 \%$ of the combined individual and parent/family programs identified in the review were found to be effective, as well as $50 \%$ of the group-based programs, $50 \%$ of the combined individual and group-based programs, $42 \%$ of the individually delivered programs, and $40 \%$ of the family/parent interventions.

\section{Quality ratings}

Table 2 presents the quality ratings for each of the studies included in the current review. Overall, $14(50 \%)$ studies were assessed as having a 'low-risk' of bias according to their generation of the allocation sequence, and 18 (64\%) studies were considered 'low-risk' for their concealment of the allocation sequence. In terms of knowledge of condition allocation, as assessed by the masking of assessors, 13 $(46 \%)$ studies were deemed as having a 'low-risk' of bias and $23(82 \%)$ studies were assessed as having a 'low-risk' of bias in their treatment of incomplete data. Eight studies (29\%) received a 'low-risk' rating on all four criteria. Of these studies, four $(50 \%)$ were found to have a significant intervention effect on suicidal ideation, suicide attempts, deliberate self-harm (DSH), and/or suicidality at immediate post-intervention or follow-up.

\section{Discussion}

The studies identified in this review of psychosocial interventions for youth suicide were predominantly face-to-face interventions delivered to mid-adolescent females with a history of suicidal ideation or attempts, and compared to a treatment as usual control condition. Intervention setting, content, delivery format (individual vs. family vs. group), and leaders were varied across programs. Very few distal interventions were identified in the review. Given the appeal and reach of new technologies among young people, and their ability to overcome some of the access, stigma and cost barriers associated with face-to-face services, this may be an area for further program development and evaluation.

Overall, just over half of the programs identified in the review reported significant effects on suicidal ideation, suicide attempts or deliberate self-harm. Small to large effect sizes were reported by the effective programs, with short and longer-term effects evident. Some of the programs that reported non-significant results had good sized effects. Given the small samples size of some of these studies, it is possible that these programs were effective, but that the trial was underpowered due to poor study recruitment or drop-out. This highlights the importance of sufficiently powering studies to detect expected intervention effects.

The current review provides preliminary evidence for the implementation of psychosocial interventions in school, community and healthcare settings. Programs in all of these settings were found to be effective for suicidal ideation and attempts, with schools showing particular promise in this population. Given the reach of schools, and the captive audience they provide, this may be a good environment in which to promote and target suicide prevention and early intervention programs with young people. In terms of program content, the current review also found a diverse range of effective interventions, with no clear stand out intervention approach. This finding reflects in part the limited evidence-base that currently exists for suicide prevention programs in this population and the need for further research to identify the most efficacious approaches to this problem. It also lends support for multi-faceted approaches to suicide prevention.

The vast majority of the effective programs identified in this review were delivered to an indicated population, suggesting that it is most effective to intervene with those exhibiting early symptoms. However, when the number of effective programs is considered in light of the total number of interventions of each type reviewed, the conclusion is quite different. When compared proportionally, $100 \%$ of the universal programs were effective, whereas only $50 \%$ of the selective and indicated interventions were effective. This suggests that both universal and targeted interventions can be effective, depending on the program delivered, and that both approaches should be considered in the prevention of suicide in this population. With only two universal programs identified in this review, there is also a need to further explore universal programs in this population.

The current review also found that programs delivered to individuals alone only had effects on suicidal ideation, while group and family programs only had effects on 
Table 2 Quality ratings for included studies

\begin{tabular}{|c|c|c|c|c|}
\hline Trial & Allocation sequence & Allocation concealment & Knowledge of allocation & Incomplete data addressed \\
\hline Randell et al. [24]; Eggert et al. [25] & $?$ & $\checkmark$ & $?$ & $x$ \\
\hline Thompson et al. [26] & $?$ & $\checkmark$ & $?$ & $\checkmark$ \\
\hline Hooven et al. [27] & $?$ & $?$ & $?$ & $\checkmark$ \\
\hline Aseltine et al. [28] & $\checkmark$ & $\checkmark$ & $?$ & $\checkmark$ \\
\hline Aseltine et al. [29] & $\checkmark$ & $\checkmark$ & $?$ & $\checkmark$ \\
\hline Tang et al. [30] & $?$ & $\checkmark$ & $\checkmark$ & $?$ \\
\hline King et al. [31]. & $\checkmark$ & $?$ & $?$ & $\checkmark$ \\
\hline King et al. [32] & $\checkmark$ & $\checkmark$ & $\checkmark$ & $\checkmark$ \\
\hline Cotgrove et al. [33] & $?$ & $?$ & $\checkmark$ & $\checkmark$ \\
\hline Fitzpatrick et al. [34] & $?$ & $?$ & $?$ & $\checkmark$ \\
\hline Harrington et al. [35] & $\checkmark$ & $\checkmark$ & $\checkmark$ & $\checkmark$ \\
\hline Huey et al. [36] & $?^{*}$ & $?^{*}$ & $?$ & $\checkmark$ \\
\hline Robinson et al. [37]. & $\checkmark$ & $\checkmark$ & $?$ & $\checkmark$ \\
\hline Green et al. [38] & $\checkmark$ & $\checkmark$ & $\checkmark$ & $\checkmark$ \\
\hline Mehlum et al. [23] & $\checkmark$ & $\checkmark$ & $\checkmark$ & $\checkmark$ \\
\hline Rosenbaum Asarnow et al. [39] & $\checkmark$ & $\checkmark$ & $\checkmark$ & $\checkmark$ \\
\hline Rossouw et al. [40] & $\checkmark$ & $\checkmark$ & $?$ & $\checkmark$ \\
\hline Pineda et al. [41] & $\checkmark$ & $\checkmark$ & $\checkmark$ & $\checkmark$ \\
\hline Alavi et al. [42] & $?$ & $?$ & $?$ & $\checkmark$ \\
\hline Diamond et al. [43] & $\checkmark$ & $\checkmark$ & $\times$ & $\checkmark$ \\
\hline Donaldson et al. [44] & $?$ & $?$ & $?$ & $\checkmark$ \\
\hline Eskin et al. [45] & $?$ & $?$ & $?$ & $?$ \\
\hline Esposito-Smythers et al. [46] & $\checkmark$ & $\checkmark$ & $\checkmark$ & $\checkmark$ \\
\hline Hazell et al. [47] & $?$ & $\checkmark$ & $\checkmark$ & $\checkmark$ \\
\hline Ourgin et al. [48] & $\checkmark$ & $\checkmark$ & $\checkmark$ & $\checkmark$ \\
\hline Power et al. [49] & $?$ & $?$ & $\checkmark$ & $?$ \\
\hline Rudd et al. [50] & $? *$ & $?^{*}$ & $?$ & $\times$ \\
\hline Wood et al. [51] & ? & $\checkmark$ & $\checkmark$ & $\checkmark$ \\
\hline
\end{tabular}

$\checkmark$, low risk of bias; ?, unclear risk of bias; $\times$, high risk of bias; *, information not provided in the trial paper, but a reference to another publication or author communication is provided

suicide attempts. Programs that included both individual and group/family components reported effects for both suicidal ideation and attempts. This finding suggests that individual level interventions may be needed to affect change in suicidal ideation, while group interactions may facilitate changes in suicide attempts. Much more research is needed to explore this potential association and the mechanisms that might contribute to this effect.

Interestingly, only one of the studies reported positive outcome effects on more than one outcome variable. One possible explanation for this finding may be the lack of short and long-term outcome measurements in many of the studies, with studies often not including both. Those studies that found significant effects for suicidal ideation often did so at immediate post-intervention or at short-term follow-up. As such, those studies that only included longerterm follow-ups did not find effects for suicidal ideation. Similarly, those studies that just included longer-term follow-ups (16- and 18-months) tended to find effects for suicide attempts, while those without these lengthier follow-ups, or large sample sizes, did not. This finding is unsurprising and provides support for the inclusion of both short and longer-term follow-ups in suicide prevention research.

The quality of the trials included in the current review did vary, with only $29 \%$ of studies receiving a 'low-risk' rating on all four of the criteria assessed. Of these studies, $50 \%$ reported significant intervention effects at post-intervention and/or follow-up. This equates to a similar proportion of studies that were found to be effective in the current review, suggesting that study quality did not significantly affect intervention outcomes. Studies that did not receive a 'low-risk' rating on a particular criterion often received an 'unclear risk of bias' rating, resulting from insufficient information being provided in the paper. This suggests that there may be a quality of reporting issue present, in which 
authors are failing to report all details of their trial, rather than these studies necessarily being of poor methodological quality. It is therefore important for study authors to include all details of their trials in outcome papers, particularly details of the randomisation process, to enable an accurate assessment of study quality to be made.

There are some limitations to the current review that should be acknowledged. This review excluded studies that did not include explicit suicide outcome measures, but may have had positive effects on other important and related factors, such as help-seeking behaviour, attitudes and literacy. It is also possible that some studies were not captured by our search strategy and therefore not identified in our review. As such, there may be other approaches to suicide prevention not identified in the current review. Another limitation of this review is that we were unable to conduct a meta-analysis due to the vast differences in participant characteristics, interventions and measurement of outcomes. The measurement of suicidal ideation, suicide attempts and deliberate self-harm differed widely among studies. Some studies employed one-item self-report measures, while others used multi-items scales or collected hospital attendance data. As a result, the quality of the collected data may vary between studies.

There are a number of critical considerations emerging from this review. First, all but one of the studies identified found a significant positive or null effect of the intervention tested. This suggests that collectively psychosocial interventions for youth suicide are safe and are unlikely to do harm. As such, the continued implementation and evaluation of these programs in the community should be encouraged, with a focus on training key personnel in schools and healthcare settings to deliver and support these interventions. The results of this review also suggest that different types of interventions delivered in a range of settings can be effective. This finding lends support to the implementation and evaluation of multimodal interventions, in which a suite of programs are delivered simultaneously in a community, to bolster prevention effects. Such interventions have been found to be effective with adults [52].

In terms of research, there is a need to strengthen the evidence-base of the programs that are currently available. This includes the targeted evaluation of programs with male adolescents who were underrepresented in a number of the trials identified, and a focus on increasing study sample size and the inclusion of short- and long-term follow-ups where possible. The further development and evaluation of universal prevention programs in schools may also be a promising avenue to explore. This assertion is supported by a recent finding of the large-scale Saving and Empowering Young Lives in Europe (SEYLE) study, which found a universal school-based intervention to have a significant positive effect on severe suicidal ideation and incident suicide attempts at 12-month follow-up [53]. Finally, with an increasing need to deliver cost-effective programs, it is important that cost and benefit analyses are included in future evaluations of youth suicide prevention interventions and that programs are evaluated in 'real world' settings outside of the confines of an RCT.

Acknowledgments ALC and $\mathrm{HC}$ are supported by National Health and Medical Research Council (NHMRC) Fellowships 1013199 and 1056964. The database used in the current review is maintained by the NHMRC Centre of Research Excellence in Suicide Prevention (1042580). We would like to thank Jacqueline Brewer, John Gosling, Katherine Petrie, Daniela Solomon, Kanupriya Kahlia Hehir and Angeline Tjhin for their assistance in completing the review.

\section{Compliance with ethical standards}

Ethical standards The manuscript does not contain clinical studies or patient data.

Conflict of interest The authors declare that they have no conflict of interest.

\section{References}

1. Patton GC, Coffey C, Sawyer SM, Viner RM, Haller DM, Bose K, Vos T, Ferguson J, Mathers CD (2009) Global patterns of mortality in young people: a systematic analysis of population health data. Lancet 374:881-892

2. Australian Bureau of Statisitics (2014) Causes of death, 2012. Cat. no. 3303.0. ABS, Canberra, Australia

3. National Center for Health Statistics (2014) Health, United States, 2013: with specific feature on prescription drugs. NCHS, Hyattsville

4. Evans E, Hawton K, Rodham K, Deeks J (2005) The prevalence of suicidal phenomena in adolescents: a systematic review of population-based studies. Suicide Life Threat Behav 35:239-250

5. Whitlock J, Wyman PA, Moore SR (2014) Connectedness and suicide prevention in adolescents: pathways and implications. Suicide Life Threat Behav 44:246-272

6. Gould MS, Greenberg T, Velting DM, Shaffer D (2003) Youth suicide risk and preventive interventions: a review of the past 10 years. J Am Acad Child Adolesc Psychiatry 42:386-405

7. Katz C, Bolton S-L, Katz LY, Isaak C, Tilston-Jones T, Sareen J, Swampy Cree Suicide Prevention Team (2013) A systematic review of school-based suicide prevention programs. Depress Anxiety 30:1030-1045

8. Gould MS, Marrocco FA, Hoagwood K, Kleinman M, Amakawa L, Altschuler E (2009) Service use by at-risk youth after schoolbased suicide screening. J Am Acad Child Adolesc Psychiatry 48:1193-1201

9. Pisani AR, Schmeelk-Cone K, Gunzler D, Petrova M, Goldston DB, Tu X, Wyman PA (2012) Assoications between suicidal high school students' help-seeking and their attitudes and perceptions of social environment. J Youth Adolesc 41:1312-1324

10. Schmeelk-Cone K, Pisani AR, Petrova M, Wyman PA (2012) Three scales assessing high school students' attitudes and perceived norms about seeking adult help for distress and suicide concerns. Suicide Life Threat Behav 42:157-172

11. Mrazek PJ, Haggerty RJ (1994) Reducing risks for mental disorders: frontiers for preventive intervention research. National Academy Press, Washington 
12. Silverman MM, Maris RW (1995) The prevention of suicidal behaviors: an overview. Suicide Life Threat Behav 25:10-21

13. Robinson J, Cox G, Malone A, Williamson M, Baldwin G, Fletcher K, O'Brien M (2013) A systematic review of schoolbased interventions aimed at preventing, treating and responding to suicide-related behavior in young people. Crisis 34:164-182

14. Brent DA, McMakin DL, Kennard BD, Goldstein TR, Mayes TL, Douaihy AB (2013) Protecting adolescents from self-harm: a critical review of intervention studies. J Am Acad Child Adolesc Psychiatry 52:1260-1271

15. Glenn CR, Franklin JC, Nock MK (2015) Evidence-based psychosocial treatments for self-injurious thoughts and behaviours in youth. J Clin Child Adolesc Psychol 44:1-29

16. Ougrin D, Tranah T, Stahl D, Moran P, Asarnow JR (2015) Therapeutic interventions for suicide attempts and self-harm in adolescents: systematic review and meta-analysis. J Am Acad Child Adolesc Psychiatry 54:97-107

17. Christensen H, Calear AL, Van Spijker B, Gosling J, Petrie K, Donker T, Fenton K (2014) Psychosocial interventions for suicidal ideation, plans, and attempts: a database of randomised controlled trials. BMC Psychiatry 14:86

18. Joiner TE, Van Orden KA, Witte TK, Selby EA, Ribeiro JD, Lewis R, Rudd MD (2009) Main predictions of the interpersonal-psychological theory of suicidal behavior: empirical tests in two samples of young adults. J Abnorm Psychol 118(3):634-646

19. Nock MK, Hwang I, Sampson NA, Kessler RC (2009) Mental disorders, comorbidity and suicidal behavior: results from the national comorbidity survey replication. Mol Psychiatry $15: 868-876$

20. Cohen JD (1988) Statistical power analysis for the behavioral sciences. Lawrence Earlbaum Associates, Hillsdale

21. Neil AL, Christensen H (2009) Efficacy and effectiveness of school-based prevention and early intervention programs for anxiety. Clin Psychol Rev 29:208-215

22. Cuijpers P, Weitz E, Twisk J, Kuehner C, Cristea I, David D, DeRubeis RJ, Dimidjian S, Dunlop BW, Faramarzi M, Hegerl U, Jarrett RB, Kennedy SH, Kheirkhah F, Mergl R, Miranda J, Mohr DC, Segal ZV, Siddique J, Simons AD, Vittengl JR, Hollon SD (2014) Gender as predictor and moderator of outcome in cognitive behavior therapy and pharmacotherapy for adult depression: an "individual patient data" meta-analysis. Depress Anxiety 31(11):941-951

23. Mehlum L, Tormoen AJ, Ramberg M, Haga E, Diep LM, Laberg S, Larsson BS, Stanley BH, Miller AL, Sund AM, Groholt B (2014) Dialectical behavior therapy for adolescents with repeated suicidal and self-harming behavior: a randomized trial. J Am Acad Child Adolesc Psychiatry 53(10):1082-1091

24. Randell BP, Eggert LL, Pike KC (2001) Immediate post intervention effects of two brief youth suicide prevention interventions. Suicide Life Threat Behav 31:41-61

25. Eggert LL, Thompson EA, Randell BP, Pike KC (2002) Preliminary effects of brief school-based prevention approaches for reducing youth suicide-risk behaviors, depression, and drug involvement. J Child Adolesc Psychiatr Nurs 15:48-64

26. Thompson EA, Eggert LL, Randell BP, Pike KC (2001) Evaluation of indicated suicide risk prevention approaches for potential high school dropouts. Am J Public Health 91:742-752

27. Hooven C, Walsh E, Pike KC, Herting JR (2012) Promoting CARE: including parents in youth suicide prevention. Fam Community Health 35:225-235

28. Aseltine RH Jr, DeMartino R (2004) An outcome evaluation of the SOS suicide prevention program. Am J Public Health 94:446-451
29. Aseltine RH Jr, James A, Schilling EA, Glanovsky J (2007) Evaluating the SOS suicide prevention program: a replication and extension. BMC Public Health 7:161

30. Tang TC, Jou SH, Ko CH, Huang SY, Yen CF (2009) Randomized study of school-based intensive interpersonal psychotherapy for depressed adolescents with suicidal risk and parasuicide behaviors. Psychiatry Clin Neurosci 63:463-470

31. King CA, Kramer A, Preuss L, Kerr DC, Weisse L, Venkataraman S (2006) Youth-Nominated Support Team for Suicidal Adolescents (Version 1): a randomized controlled trial. J Consult Clin Psychol 74:199-206

32. King CA, Klaus N, Kramer A, Venkataraman S, Quinlan P, Gillespie B (2009) The Youth-Nominated Support Team-Version II for suicidal adolescents: a randomized controlled intervention trial. J Consult Clin Psychol 77(5):880-893

33. Cotgrove A, Zirinsky L, Black D, Weston D (1995) Secondary prevention of attempted suicide in adolescence. J Adolesc 18:569-577

34. Fitzpatrick KK, Witte TK, Schmidt NB (2005) Randomized controlled trial of a brief problem-orientation intervention for suicidal ideation. Behav Ther 36:323-333

35. Harrington R, Kerfoot M, Dyer E, McNiven F, Gill J, Harrington V, Woodham A, Byford S (1998) Randomized trial of a home-based family intervention for children who have deliberately poisoned themselves. J Am Acad Child Adolesc Psychiatry 37:512-518

36. Huey SJ Jr, Henggeler SW, Rowland MD, Halliday-Boykins CA, Cunningham PB, Pickrel SG, Edwards J (2004) Multisystemic therapy effects on attempted suicide by youths presenting psychiatric emergencies. J Am Acad Child Adolesc Psychiatry 43:183-190

37. Robinson J, Yuen HP, Gook S, Hughes A, Cosgrave E, Killackey E, Baker K, Jorm A, McGorry P, Yung A (2012) Can receipt of a regular postcard reduce suicide-related behaviour in young help seekers? A randomized controlled trial. Early Interv Psychiatry 6:145-152

38. Green J, Wood A, Kerfoot M, Trainor G, Roberts C, Rothwell J, Woodham A, Ayodeji E, Barrett B, Byford S (2011) Group therapy for adolescents with repeated self harm: randomised controlled trial with economic evaluation. BMJ 342:d682

39. Rosenbaum Asarnow J, Baraff LJ, Berk M, Grob CS, DevichNavarro M, Suddath R, Piacentini JC, Rotheram-Borus MJ, Cohen D, Tang L (2011) An emergency department intervention for linking pediatric suicidal patients to follow-up mental health treatment. Psychiatr Serv 62:1303-1309

40. Rossouw TI, Fonagy P (2012) Mentalization-based treatment for self-harm in adolescents: a randomized controlled trial. J Am Acad Child Adolesc Psychiatry 51:1304-1313

41. Pineda J, Dadds MR (2013) Family intervention for adolescents with suicidal behavior: a randomized controlled trial and mediation analysis. J Am Acad Child Adolesc Psychiatry 52:851-862

42. Alavi A, Sharifi B, Ghanizadeh A, Dehbozorgi G (2013) Effectiveness of cognitive-behavioral therapy in decreasing suicidal ideation and hopelessness of the adolescents with previous suicidal attempts. Iran J Pediatr 23:467-472

43. Diamond GS, Wintersteen MB, Brown GK, Diamond GM, Gallop R, Shelef K, Levy S (2010) Attachment-based family therapy for adolescents with suicidal ideation: a randomized controlled trial. J Am Acad Child Adolesc Psychiatry 49:122-131

44. Donaldson D, Spirito A, Esposito-Smythers C (2005) Treatment for adolescents following a suicide attempt: results of a pilot trial. J Am Acad Child Adolesc Psychiatry 44:113-120

45. Eskin M, Ertekin K, Demir H (2008) Efficacy of a problem-solving therapy for depression and suicide potential in adolescents and young adults. Cognit Ther Res 32:227-245 
46. Esposito-Smythers C, Spirito A, Kahler CW, Hunt J, Monti P (2011) Treatment of co-occurring substance abuse and suicidality among adolescents: a randomized trial. J Consult Clin Psychol 79:728-739

47. Hazell PL, Martin G, McGill K, Kay T, Wood A, Trainor G, Harrington R (2009) Group therapy for repeated deliberate self-harm in adolescents. J Am Acad Child Adolesc Psychiatry 48:662-670

48. Ougrin D, Boege I, Stahl D, Banarsee R, Taylor E (2013) Randomised controlled trial of therapeutic assessment versus usual assessment in adolescents with self-harm: 2-year follow-up. Arch Dis Child 98:772-776

49. Power P, Bell R, Mills R, Herrman-Doig T, Davern M, Henry L, Yuen H, Khademy-Deljo A, McGorry P (2003) Suicide prevention in first episode psychosis: the development of a randomised controlled trial of cognitive therapy for acutely suicidal patients with early psychosis. Aust N Z J Psychiatry 37:414-420

50. Rudd MD, Rajab MH, Orman DT, Stulman DA, Joiner T, Dixon W (1996) Effectiveness of an outpatient intervention targeting suicidal young adults: preliminary results. J Consult Clin Psychol 64:179-190
51. Wood A, Trainor G, Rothwell J, Moore A, Harrington R (2001) Randomized trial of group therapy for repeated deliberate selfharm in adolescents. J Am Acad Child Adolesc Psychiatry 40:1246-1253

52. Székely A, Thege BK, Mergl R, Birkás E, Rózsa S, Purebl G, Hegerl U (2013) How to decrease suicide rates in both genders? An effectiveness study of a community-based intervention (EAAD). PLoS One 8:e75081

53. Wasserman D, Hoven CW, Wasserman C, Wall M, Eisenberg R, Hadlaczky G, Kelleher I, Sarchiapone M, Apter A, Balazs J, Bobes J, Brunner R, Corcoran P, Cosman D, Guillemin F, Haring C, Iosue M, Kaess M, Kahn J-P, Keeley H, Musa GJ, Nemes B, Postuvan V, Saiz P, Reiter-Theil S, Varnik A, Varnik P, Carli V (2015) School-based suicide prevention programmes: the SEYLE cluster-randomised, controlled trial. Lancet 385:1536-1544 